\title{
sciendo
}

\section{Differences in Trunk Strength Between Weightlifters and Wrestlers}

\author{
by \\ Amira Ben Moussa Zouita1, Sghaier Zouita ${ }^{1,2}$, Catherine Dziri³, Matt Brughellit, \\ David G. Behm ${ }^{5}$, Anis Chaouachi'2,4,6
}

\begin{abstract}
Investigations of trunk strength with high-level athletes are limited. The purpose of this study was to compare maximal concentric isokinetic trunk extension and flexion torque, power, and strength ratios between high-level weightlifters $(n=20)$, wrestlers $(n=20)$ and a control $(n=25)$ population. Isokinetic dynamometry was used to evaluate peak torque, power and strength ratios during seated trunk extension/flexion actions at $60 \%$ s and $180 \%$. There were no significant anthropometric differences between groups. Overall, trunk isokinetic force variables as a function of the increase in angular velocity, showed a decrease in peak torque, but an increase in power (athletes and controls). Compared to the control group, athletes demonstrated significantly higher trunk extension torque $(+67.05 \mathrm{~N} \cdot \mathrm{m}, E S=0.81)$ and power $(+49.28 \mathrm{~N} \cdot \mathrm{m}, \mathrm{ES}=0.82)$ at $60 \% \mathrm{~s}$ and $180 \%$, respectively. Athletes produced significantly greater trunk flexionextension ratios at $60 \%$ and $180 \%(E S=0.80-0.47)$ than controls. Weightlifters and wrestlers exhibited significantly higher extensor than flexor torque at all angular velocities. Weightlifters demonstrated greater torque $(E S=0.79)$ than wrestlers at $60 \%$ s. The wrestlers' average power was significantly higher $(E S=0.43)$ than weightlifters at $180 \%$. There were no significant ratio differences between wrestlers (66.23\%) and weightlifters (72.06\%). Weightlifters had stronger extensor muscles at $60 \%$, whereas wrestlers had higher power at $180 \%$ for extensor muscles. It was postulated that the extensor muscles were stronger than the flexors to ensure trunk stabilisation, and for prevention of injuries. These differences seem to be associated to the movements that occur in each sport in terms of both muscle actions and contractile forces.
\end{abstract}

Key words: isokinetic dynamometry, sport performance, spine, core, back.

\section{Introduction}

Trunk muscles are important for general activities and all sports that require abdominal and back strength for stability (Behm et al., 2010a, b) and in injury prevention related to health, rehabilitation (Behm et al., 2011; Behm and Colado, 2012) and physical performance (Behm and Colado, 2013; Maszczyk et al., 2016; Stastny et al., 2017; Steele et al., 2014). Trunk strength capacity is considered essential to compensate for external forces and loads in adult athletes (Kibler et al., 2006), which are dependent on gender, age, and specific sports (Skrzek et al., 2012). Repetitive loading with high components of translation and rotation is believed to result in high impact forces (Adirim and Cheng, 2003; Gołaś et al., 2016; Jones et al., 2005; Muller et al., 2017; Sassmannshausen and Smith, 2002). Weightlifters and wrestlers need a highly developed strength capacity of abdominal

1 - Higher Institute of Sport and Physical Education, Ksar-said, University of Manouba, Tunis, Tunisia.

2 - Tunisian Research Laboratory "Sports Performance Optimization", National Center of Medicine and Science in Sports (CNMSS), Tunis, Tunisia.

3 - Department of Medicine Physical and Functional Rehabilitation of the National Institute of Orthopedics "M.T. Kassab" Tunisia.

4 - AUT University, Sports Performance Research Institute NewZealand, Auckland, New Zealand.

5 - School of Human Kinetics and Recreation, Memorial University of Newfoundland, St. John's, Newfoundland, Canada. 6 - PVF Football Academy, Hang Yen, Vietnam. 
and back muscles to compensate for these sportspecific loads (Baur et al., 2010). In general, athletes show higher trunk extensor strength values (Yahia et al., 2011) with smaller flexion-extension ratios of trunk peak torque (Mueller et al., 2011).

Weightlifting is a sport where two lifts are contested. They all involve the use of explosive extensions of the ankles, knees, and hip joints in their proper sequence. In the final extension of the snatch or clean and the drive in the jerk, the bar speed must be even greater in order to drive it all the way to full extension (Fry et al., 2006). Two of the major factors that enable the athlete to maintain the weight in the proper position overhead are (a) trunk balance and stability between the flexors and extensors, and (b) effective deceleration throughout the anterior chain trunk muscles. This will allow the athlete to stop the weight in the proper position. To provide the most solid position for a weight that is being lifted, a strong base of support between the abdominals and the low back muscles should be a priority for long-term success and health of the athlete (Hendrick and Wada, 2008). Hence, it is suggested that athletes should strengthen the explosive force of the trunk extensors. Weightlifting generates a high level of muscular power effectively transfering power to the bar in a short time. The snatch is a characteristic movement of maximum power in which speed and coordination play a decisive role (Hendrick and Wada, 2008).

Similarly to weightlifters, wrestlers present high absolute maximal trunk extensor strength (Kolber and Beekhuizen, 2007). A wrestling match is characterized by sudden explosive attacks and counterattacks in order to lift the opponent powerfully to the mat and escape from the bottom position. Generally, explosive strength is of great importance in wrestling.

Preliminary results from Soldati et al. (1992) emphasized the high levels of extensor muscle strength for groups of weightlifters and wrestlers compared with a sedentary population, but similar flexor muscle strength were found between the athletic groups and the sedentary population. However, few previous investigations had directly explored the impact of angular velocity on trunk flexion and extension strength and ratios. Also, there is a lack of data concerning the interpretation of spinal isokinetic variables.

Presently, isokinetic testing of trunk extensor and flexor strength is the standard measure of core stability in clinical sports medicine to assess injury risk and post-operative rehabilitation (Aasa et al., 2017; Orchard et al., 2004) and to assess muscle strength capacity of athletes (Yahia et al., 2011). Isokinetic measurement has high reliability and precision (Mueller et al., 2011) and is often used for dynamic strength measurements to understand the mechanical profile of trunk muscles (Van Damme et al., 2013). Data from isokinetic trunk flexion and extension strength should help accurately predict measures of trunk performance, thereby allowing comparisons to the body of normative data. Furthermore, identification of strength deficits with and without pathologies as well as the evaluation of preventive and rehabilitative interventions is of major concern. Extrapolating muscular variables from the torque and powervelocity relationship corresponds to individual muscle characteristics, and enables athletes to be assessed for safety. Determining these variables has practical applications in general and particularly during clinical rehabilitation and scientific research applications in sports medicine (Guilhem et al., 2014).

Consequently, the purpose of this study was to assess isokinetic trunk strength performance in the sagittal plane. First, to perform a case-control investigation in order to compare maximal concentric isokinetic trunk extension and flexion torque, power, and ratios between highlevel athletes and a control population. Secondly, to detect the specific training differences in trunk strength capacity of weightlifters and wrestlers.

\section{Methods}

\section{Participants}

A case group of 40 high-level male athletes were recruited from wrestling $(n=20)$, and weightlifting $(n=20)$. Additionally, a non-athlete control group was recruited $(n=25)$ (Table 1$)$. The non-athlete group were recreationally active, but did not participate in a sport more than 2 days per week. They performed occasional physical activity, such as running or playing soccer, volleyball, or basketball, but only at a recreational level. During the study, the wrestling and weightlifting athletes were preparing for the upcoming national championship and international competitions following a periodized strength and 
conditioning program. Wrestling training was directed to develop anaerobic power and capacity, aerobic power, maximal dynamic and isometric strength, explosive strength, and strength endurance using general guidelines from which to build a highly integrated wrestling program (Chaabene et al., 2017; Kraemer et al., 2014; Murlasits, 2004). The weightlifting training program focused on the two main Olympic lifts and their derivatives with similar multi-joint movements, back and front squats, and major pushing and pulling movements, in addition to metabolic conditioning (Storey and Smith, 2012). Complementary exercises with movement patterns similar to the competitive lifts (e.g. hang/power snatch, hang/power clean, snatch and clean pulls, front and back squats) and supplementary exercises (e.g. overhead presses, back extensions and abdominal work) that target synergistic muscle groups were also used (Storey and Smith, 2012). Trunk or torso training was addressed as a separate component for all athletes to provide additional work for the abdominal and lower back muscles. Subjects suffering from low back pain during the familiarization process with the dynamometer were excluded. All subjects provided informed consent for participation in the research study. The study was conducted according to the Declaration of Helsinki, and the protocol was fully approved by the Ethics Committee of the National Center of Medicine and Science of Sports of Tunis (CNMSS) before the commencement of the assessments.

Initially, a medical check-up was performed to confirm that all participants were free from injury and of adequate health to perform strength testing. Participants were instructed not to eat for at least 3 hours and not to drink coffee or beverages containing caffeine for at least 8 hours before each testing session. No participant was suffering from lower extremity muscular injury at the time of testing or during 6 months before testing (Grabiner and Jeziorowski, 1992). Subsequently, anthropometric data were recorded, including body mass and body height. Prior to trunk strength testing, the participants performed a general warm-up, comprising 10 minutes of running at a self-selected speed on a treadmill. Since the participants had no previous experience with isokinetic trunk strength testing, the protocol began with a familiarization trial of isokinetic trunk flexion and extension at a moderate intensity (Recio et al., 2017). A Biodex ${ }^{\circledR}$ dynamometer system 3 was used to measure the isokinetic muscular functions of the trunk.

For the trunk strength measurement protocol, participants were tested in a seated position with their anterior superior iliac spines (ASIS) in alignment with the fixed axis of rotation of the Biodex unit. The reliability of the Biodex System 3 has previously been assessed (Secchi et al., 2010). From a practical standpoint, muscle assessment methods using an isokinetic dynamometer are considered to be valid and very reliable, with correlation coefficients of $0.93-0.99$ for peak force values and between $0.91-0.96$ for total workload values (Guilhem et al., 2014).

The height of the foot plates was adjusted to provide $15^{\circ}$ of knee flexion and the sacral pad was subsequently placed to maintain alignments of the axis of rotation. Four stabilization straps were placed across each participant. One strap was placed over both hips and one across the proximal thighs. The range of flexion and extension was $50^{\circ}$ (Guilhem et al., 2014). The participant was instructed to grasp the shoulder straps during testing.

The warm-up and familiarization trial consisted of one set of 10 consecutive, submaximal concentric trunk flexion and extension repetitions at $120 \%$ s with a 1-min rest interval between sets. Each repetition started from the trunk flexion position. After the warm-up and familiarization, participants performed maximum concentric isokinetic trunk flexion and trunk extension for five repetitions at $60 \% \mathrm{~s}$ and for 15 repetitions at $180^{\circ} \%$. Verbal encouragement was given throughout the entire test to ensure participants' maximum effort. Peak torque of trunk flexion and trunk extension $(\mathrm{N} \cdot \mathrm{m})$, mean power (watts) and the ratio (\%) of flexion to extension torque were calculated.

\section{Statistical Analysis}

Statistical analysis was performed with descriptive measures (mean \pm standard deviation (SD)) for all strength measures. To make sure that the data was normally distributed, a KolmogorovSmirnov test was applied. The T-test and one-way variance analysis and the Tukey's post hoc test for the mean differences between different sports were used. All the tests performed were calculated with SPSS. 20 software on the basis of objectives and the 
significance level was set at $p<0.05$. Effect size (Cohen's d) for each significant finding was calculated (Cohen, 1998; Lalongo, 2016).

\section{Results}

The main anthropometric characteristics of the sample population according to the sport specialty and control group are presented in Table 1. There were no significant anthropometric differences between the athletes (wrestlers and weightlifters) and the control group.

The trunk isokinetic force variables as a function of the increase in angular velocity, showed a decrease in peak torque, but an increase in power for the athlete and control groups (Table 2). Compared to the control group, the athletes, as a combined group, demonstrated significantly higher trunk extension torque $(+67.05 \mathrm{~N} \cdot \mathrm{m}$,
$\mathrm{ES}=0.81, p<0.05)$ and power $(+49.28 \mathrm{~N} \cdot \mathrm{m}, \mathrm{ES}=0.82$, $p<0.05)$ at $60^{\circ} / \mathrm{s}$ and $180^{\circ} / \mathrm{s}$, respectively. Thus, the flexion to extension ratio of the athletes was significantly lower than for the control group (Table 2). The ratio of the control group decreased with increased velocity (Table 2).

Insert Table 2: Peak torque, power and ratio of trunk extension and flexion at each angular velocity of each group

Weightlifters and wrestlers exhibited significantly higher extensor than flexor torque at all angular velocities $(p<0.05)$. Weightlifters were significantly stronger (greater torque) $(+103.13$ $\mathrm{N} \cdot \mathrm{m}, \mathrm{ES}=0.79, p<0.05)$ than wrestlers at $60 \% \mathrm{~s}$ (Figure 1). The wrestlers' average power was significantly higher $(+43.35$ Watts, ES $=0.43, p<$ 0.05 ) than that of weightlifters at $180 \%$ s (Figure 2 ). There were no significant ratio differences between wrestlers $(66.23 \%)$ and weightlifters $(72.06 \%)$ (Table 2).

Table 1

Anthropometric characteristics of study participants.

\begin{tabular}{cccc}
\hline & Age (years) & Body mass $(\mathrm{kg})$ & Body height $(\mathrm{m})$ \\
\hline Controls $n=\mathbf{2 5}$ & $21.2 \pm 0.3$ & $74.6 \pm 1.2$ & $173.5 \pm 2.1$ \\
Wrestlers $n=\mathbf{2 0}$ & $21.5 \pm 3.6$ & $75.5 \pm 5.7$ & $175 \pm 6.0$ \\
Weightlifters $\boldsymbol{n}=\mathbf{2 0}$ & $21.1 \pm 4.07$ & $73.8 \pm 4.9$ & $176.5 \pm 7.0$ \\
\hline
\end{tabular}


Table 2

Peak torque, power and ratio of trunk extension and flexion at each angular velocity of the athletes and the control group.

\begin{tabular}{|c|c|c|c|c|}
\hline \multirow[b]{3}{*}{ Groups } & \multicolumn{2}{|c|}{ Extension } & \multicolumn{2}{|c|}{ Flexion } \\
\hline & \multicolumn{4}{|c|}{ Peak Torque $(N \cdot m)$} \\
\hline & $60^{\circ} / \mathrm{s}$ & $180^{\circ} / \mathrm{s}$ & $60^{\circ} / \mathrm{s}$ & $180^{\circ} / \mathrm{s}$ \\
\hline & 440.05 & 345.26 & 297.34 & 211.98 \\
\hline \multirow[t]{2}{*}{ Athletes } & $\pm 66.24 * \S$ & $\pm 64.57^{*}$ & \pm 50.06 & \pm 68.81 \\
\hline & 373.01 & 344.16 & 249.23 & 190.10 \\
\hline \multirow[t]{3}{*}{ Control } & $\pm 68.12^{*}$ & $\pm 70.53^{*}$ & \pm 50.03 & \pm 42.23 \\
\hline & \multicolumn{4}{|c|}{ Power (Watt) } \\
\hline & 155.18 & 280.50 & 109.37 & 158.47 \\
\hline \multirow[t]{2}{*}{ Athletes } & $\pm 42.95 *$ & $\pm 60.34^{*} \S$ & \pm 21.40 & \pm 20.32 \\
\hline & 126.64 & 231.22 & 86.65 & 136.16 \\
\hline \multirow[t]{3}{*}{ Control } & $\pm 44.87^{*}$ & $\pm 59.38^{*}$ & \pm 27.91 & \pm 38.52 \\
\hline & \multicolumn{4}{|c|}{ Ratio (\%) } \\
\hline & \multicolumn{2}{|c|}{67.56} & \multicolumn{2}{|c|}{61.39} \\
\hline \multirow[t]{2}{*}{ Athletes } & \multicolumn{2}{|c|}{ $\pm 14.24 \S$} & \multicolumn{2}{|c|}{ $\pm 16.32 \S$} \\
\hline & \multicolumn{2}{|c|}{56.81} & \multicolumn{2}{|c|}{55.23} \\
\hline Control & \multicolumn{2}{|c|}{ \pm 13.4} & \multicolumn{2}{|c|}{ \pm 13.0} \\
\hline
\end{tabular}

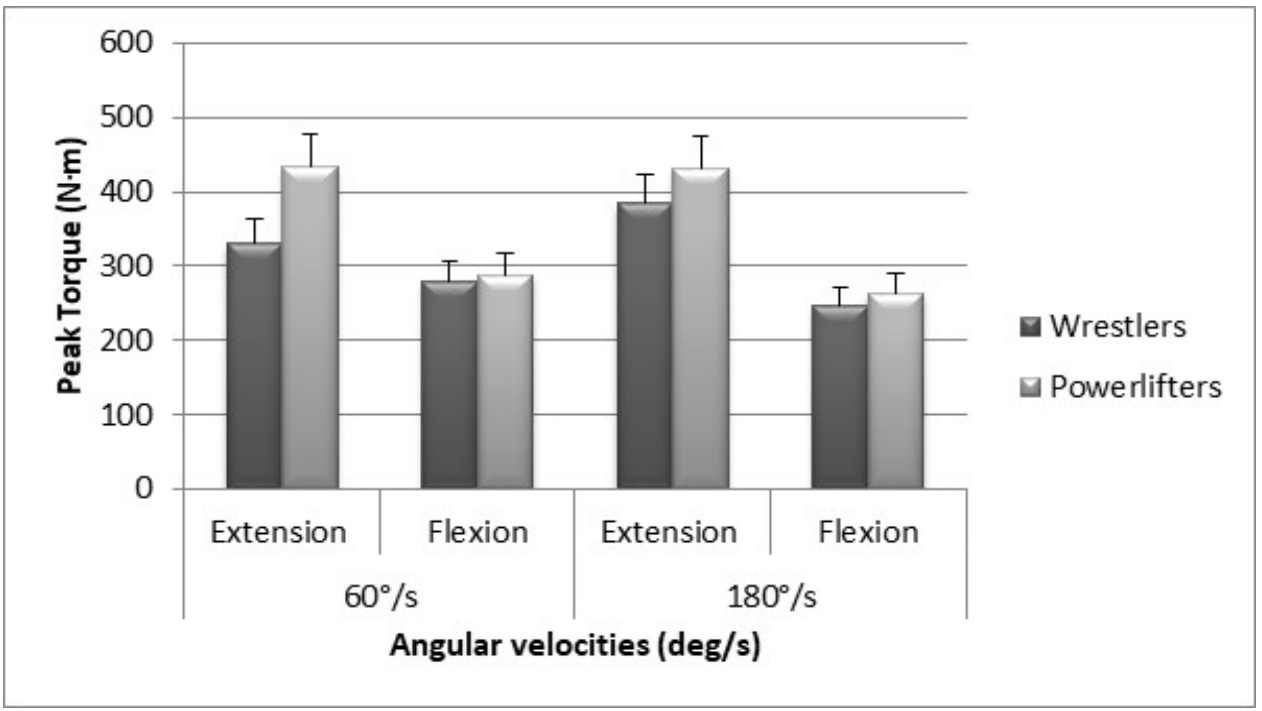

Figure 1

Mean peak torque during extension and flexor movement at 60 and $180 \%$ in wrestlers and weightlifters. Data presented as mean $\pm S D$. §: Significant differences between weightlifters and wrestlers $(p<0.05)$ 


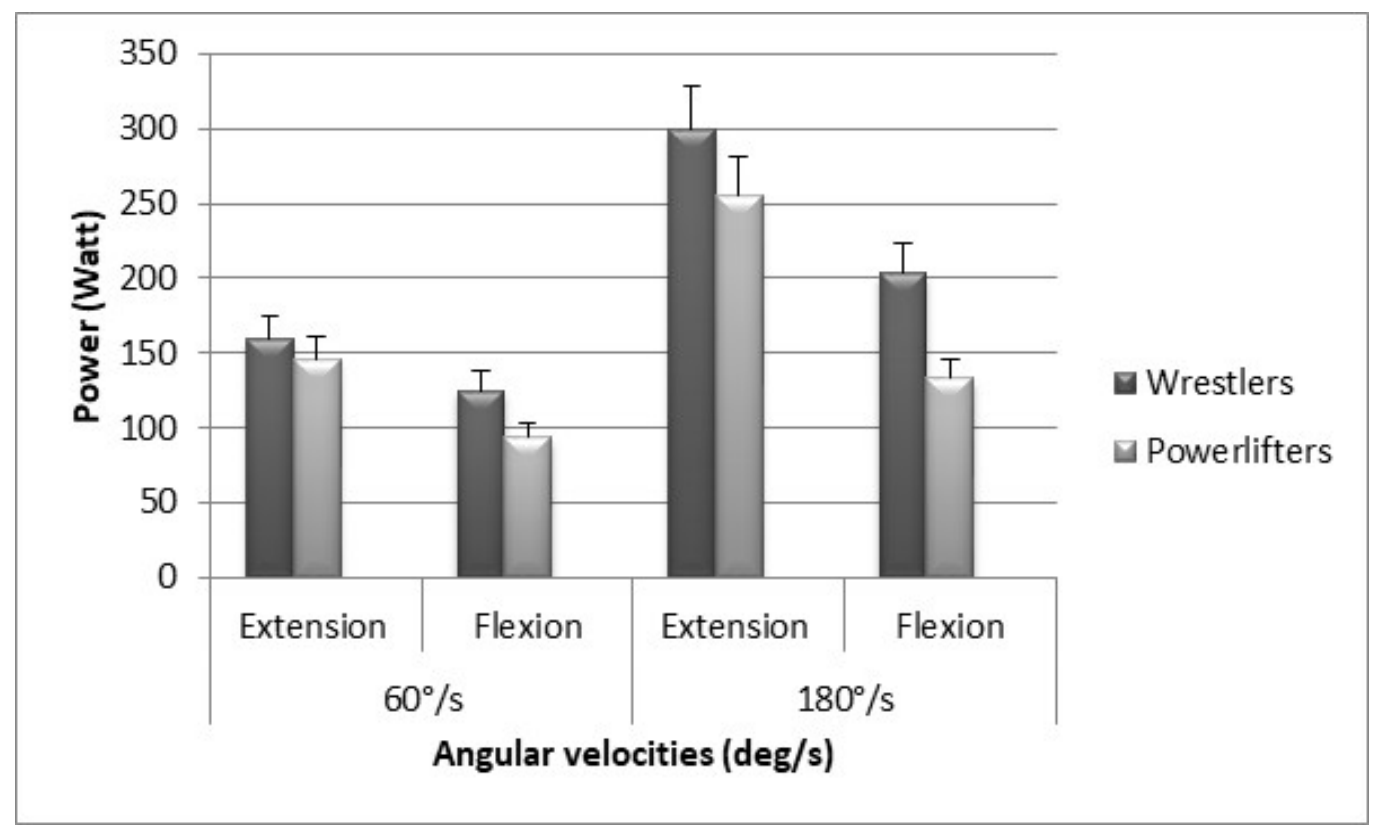

Figure 2

Mean power during extension and flexor movement at 60 and $180 \%$ in wrestlers and weightlifters. Data presented as mean $\pm S D$. §: Significant differences between weightlifters and wrestlers $(p<0.05)$

\section{Discussion}

The general objective of the study was to determine the profile of trunk isokinetic strength among wrestlers and weightlifters and to detect differences in peak torque, power and the ratio of flexor/extensor torque. The main findings illustrated trends for increased peak torque and power of extensor compared to flexor muscles for both groups $(p<0.05)$. The effect was more marked for trunk extension in the athlete group than in the active control group at $60^{\circ} \mathrm{s}$. This result shows the importance of trunk muscles for providing spinal stability depending on the relative activation of all trunk muscles (Cholewicki and VanVliet, 2002). The back extensors are particularly important postural muscles (Ward et al., 2009). They function to help stabilize the vertebral column; because of their anti-gravitational function, the extensors are involved in the majority of human postures and movement. Tension in the abdominal muscles may provide additional stability to the vertebral column by generating tension in the thoracolumbar fascia as well as increasing additional pressure (Yaprak, 2013).

The greater strength and power of the athletes in the present study can be correlated to the notion that certain sports may predispose athletes to action or task specific training adaptations (Behm and Sale, 1993). Physical activities often require flexibility of the spine and various activities require the ability to bend or twist the spine in order to move the upper body (Henchoz and So, 2008). This implies that a strong posterior muscle chain and a good balance between the lumbar, thoracic and hip extensors is 
crucial. Literature provides evidence that strength training of the trunk extensors in sport is important in the prevention of low back pain (LBP) (Durall et al., 2009). Exercise will lead to a decrease in pain and disability, and to a reduction of LBP occurrence among athletes (Gabriel et al., 1998; Renkawitz et al., 2006, 2008). Trunk extensors strength training is also beneficial for sport performance (Durall et al., 2009) and various types of physical activity and sports (Guzik et al., 1996) due to its relationship with core stability (Behm et al., 2010a, b; Behm and Colados, 2013). A functional activity oriented training programme improves performance in specific functional tasks (Hibbs et al., 2008).

However, few previous investigations have directly compared trunk flexion and extension strength and flexion / extension ratios between athletes. In healthy athletes, trunk strength is typically greatest in sagittal plane extension followed by sagittal plane flexion (Baur et al., 2010). Our athletes presented average extensors and flexors peak torque of $440.05 \pm 66.24$ $(\mathrm{N} \cdot \mathrm{m})$ and $345.26 \pm 64.57(\mathrm{~N} \cdot \mathrm{m})$, respectively, with a ratio of flexion to extension between $0.61 \pm 0.14$ $0.67 \pm 0.16$. These values are similar to those presented in the literature for extensor, but higher for flexor muscle groups. Several studies have assessed trunk flexion and extension torque and it is clear that athletes tend to show higher trunk strength values, but also the smallest ratio of trunk flexion to extension (Yahia et al., 2011). Elite athletes typically show a capacity of between 150 $240 \mathrm{~N} \cdot \mathrm{m}$ for trunk flexion and between $200-450$ $\mathrm{N} \cdot \mathrm{m}$ for trunk extension (Baur et al., 2010). Ratios of trunk flexion to extension in healthy athletes tend to be between $0.5-0.7$, which occurs in tandem with increased trunk extensor strength (Mueller et al., 2011). Differences in isokinetic variables with those presented in the literature may be attributed to differences in methodology and the type of the dynamometer used.

Sufficient trunk strength is a basic requirement of athletic performance and for sportspecific profiling. Thus, the second purpose of the study addressed sport-specific trunk strength training adaptations of weightlifters and wrestlers. Our results showed that weightlifters had greater extensor muscle torque (strength) at $60 \%$, whereas wrestlers had higher power output at $180^{\circ}$ /s with the trunk extensor and flexor muscles. Our findings indicated that the sport-specific characteristics of the isokinetic strength and power trunk muscles obviously differed between a combat sport (wrestling) and weightlifting (Baranto et al., 2009; Mueller et al., 2011), with weightlifters presenting greater strength measures, whereas wrestlers exhibited higher power measures.

As a result, weightlifters generate greater strength and move at slower velocities (Chiu and Schilling, 2005; Fry et al., 2003). Developing maximal strength is required and allow greater loads to be lifted by utilising the posterior-chain musculature during resistance training. This training approach is thought to be most beneficial in developing maximal muscle strength (Baechle et al., 2008; Fleck and Kraemer, 2004). In this approach, exercises are selected based on their similarity to movements that occur in the sport being trained for in terms of both muscle actions and contractile forces (action specificity: Behm and Sale, 1993).

Unfortunately, only a small number of researchers have examined dynamic muscle strength and muscle power profiles in exercises related to specific skills in wrestling (HubnerWozniak, 2004; Kraemer et al., 2001; Mirzaei et al., 2009). Wrestling has been described as an intermittent physical event, which demands strength and power of both the upper and lower body (Hubner-Wozniak, 2004; Kraemer et al., 2004). Wrestling neuromuscular performance has been previously examined during isokinetic upper and lower extremity muscle tests (Kraemer et al., 2001). Unfortunately, a small number of researchers have examined dynamic muscle strength and muscle power profiles of the trunk in wrestling (Mirzaei et al., 2009). We suppose that higher power output of the trunk will give elite wrestlers a clear advantage during most frequently used takedown techniques and during wrestling moves.

Thus, a consequence of differences in the peak torque profile between the two groups is that the flexor to extensor muscle ratio of weightlifters was significantly lower $(0.52-0.59)$ than wrestlers (0.72-0.76). Regarding the trunk muscle agonist/antagonist ratio, it is known that an imbalance in muscle strength may be a risk factor for lumbar pain (Baechle et al., 2008). The literature shows that the relationship between trunk 
flexor/extensor muscles remains between 0.71 and 0.92 for healthy athletes (Fleck and Kraemer, 2004; Garcia-Pallares et al., 2011). It is necessary to be prudent about the interpretation of the ratio as there is variability in isokinetic measurements related to the muscular group tested, the type of the athlete, the level and the type of activity. The development of normative standards and their comparison with athletes, should take into account the characteristics of the population studied (age, sex, occupation, physical activity) (Lee et al., 1999).

Differences in sport-specific training of trunk muscles resulting from the differences in trunk motion that are crucial to the two sports could explain variability in the extension/flexion trunk muscle ratios between weightlifters and wrestlers. The principle of training specificity indicates that exercise choice should match the movement patterns and muscle actions of the sport as closely as possible if one is to achieve optimal levels of transfer (Behm and Sale, 1993). As the demands of the sport determine the training program, the training program influences trunk strength in weightlifting and wrestling. Although both sports necessitate great trunk stability, the different sport-specific trunk motions involved in each sport can induce different muscular adaptations in the corresponding trunk muscles as demonstrated between wrestlers and judo athletes (Iwai et al., 2008). Regarding the trunk motions during wrestling, wrestlers are required to strengthen sagittal movements such as flexion and extension motions in order to grapple with an opponent, assume a high bridge motion, while also assuming the low posture that is unique to wrestling and to tackle and lift an opponent during practice and competitions (Iwai et al., 2008). All the aforementioned tasks or movements would require wrestlers to firmly stabilize their trunk region and produce high trunk flexion and extension force output (Iwai et al., 2008). Yet, that does not imply that wrestlers would require greater trunk flexor and extensor strength than weightlifters.

On the other hand, the training structure of competitive weightlifters is characterized by frequent use of high-intensity resistance exercises (Storey and Smith, 2012). Although the use of weightlifting exercises such as the snatch and clean and jerk is becoming increasingly popular across a number of sports, the frequency of high intensity resistance exercise performed by weightlifters is unmatched by other athletes and exceeds the American College of Sports Medicine's recommendations for strength and power training (Storey and Smith, 2012). In weightlifting training, the primary focus is on trunk strength (Hedrick, 2000). Trunk strength is critical because all movements either originate in or are coupled (transfer of force from the lower to the upper body and vice versa) through the trunk (Hedrick, 2000). An example of this is performing a clean and jerk in weightlifting. With a well-developed core, the force generated by triple extension of the ankle, knee, and hip joints transfers through the core with little energy dissipation, providing greater power potential and more efficient movement (Hedrick, 2000). The collective differences in competitive demands and the required physiological adaptations of wrestlers and weightlifters may account for these trunk strength differences.

In conclusion, it is proposed that the extensor muscles were stronger than the flexors for two reasons: 1) to ensure trunk stabilisation, and 2) for prevention of injuries. Weightlifting had stronger extensor muscles at $60 \%$, whereas wrestlers had higher power at $180 \%$ for extensor muscles. These differences seem to be associated to the movements that occur in each sport in terms of both muscle actions and contractile forces.

\section{References}

Aasa U, Svartholm I, Andersson F, Berglund L. Injuries among weightlifters and powerlifters: a systematic review. Br J Sports Med, 2017; 51: 211-219

Adirim TA, Cheng TL. Overview of injuries in the young athlete. Sports Med, 2003; 33: 75-81

Baechle TR, Earle RW, Wathen D. Resistance training. In: Essentials of Strength \& Conditioning (3rd ed.). TR Baechle and RW Earle, eds. Champaign, IL: Human Kinetics, 2008: 381-412 
Baranto A, Hellstrom M, Cederlund CG, Nyman R, Sward L. Back pain and MRI changes in the thoracolumbar spine of top athletes in four different sports: a 15-year follow-up study. Knee Surg Sports Traumatol Arthrosc, 2009; 17: 1125-1134

Baur H, Mueller S, Pilz F, Mayer P, Mayer F. Trunk extensor and flexor strength of long-distance race car drivers and physically active controls. J Sports Sci, 2010; 28(11): 1183-7

Behm DG, Sale DG. Velocity Specificity of Resistance Training: A Review. Sports Med, 1993; 15(6): 1-15

Behm DG, Colado Sanchez JC. Instability Resistance Training Across the Exercise Continuum. Sports Health, 2013; 5(6): 500-503

Behm DG, Colado Sanchez JC. The effectiveness of resistance training using unstable surfaces and devices for rehabilitation. Inter J Sports Phys Ther, 2012; 7(2): 226-241

Behm DG, Drinkwater EJ, Willardson JM, Cowley PM. Canadian Society for Exercise Physiology Position Stand on The Use of Instability to Train the Core In Athletic and Non-Athletic Conditioning. Appl Physiol, Nutr Metabol, 2010a; 35: 109-112

Behm DG, Drinkwater EJ, Willardson JM, Cowley PM. The role of instability rehabilitative resistance training for the core musculature. Strength Cond J, 2011; 33(3): 72-81

Behm DG, Drinkwater EJ, Willardson JM., Cowley PM. The use of instability to train the core musculature. Appl Physiol Nutr Metabol, 2010b; 35: 95-108

Chaabene H, Negra Y, Bouguezzi R, Mkaouer B, Franchini E, Julio U, Hachana Y. Physical and Physiological Attributes of Wrestlers: An Update. J Strength Cond Res, 2017; 31: 1411-1442

Chiu L, Schilling BK. A primer on weightlifting: From sport to sports training. Strength Cond J, 2005; 27: 42-48

Cholewicki J, VanVliet JJt. Relative contribution of trunk muscles to the stability of the lumbar spine during isometric exertions. Clin Biomech, 2002;17(2): 99-105

Cohen J. Statistical power analysis for the behavioural sciences. Hillside N.J.: L. Erbraum Associates. 1988; Chapter 1: 8-17

Durall CJ, UdermannBE, Johansen DR, Gibson B, Reineke DM, Reuteman P. The effects of preseason trunk muscle training on low-back pain occurrence in women collegiate gymnasts. J Strength Cond Res, 2009; 23: 86-92

Fleck SJ, Kraemer WJ. Advanced training strategies In: Designing Resistance Training Programs (3rd ed.). Champaign, IL: Human Kinetics, 209-239; 2004

Fry AC, Ciroslan D, Fry MD, LeRoux CD, Schilling BK, Chiu LZ. Anthropometric and performance variables discriminating elite American Junior men weightlifters. J Strength Cond Res, 2006; 20(4): 861-6

Fry AC, Schilling BK, Staron RS, Hagerman FC, Hikida RS, Thrush JT. Muscle fiber characteristics and performance correlates of male Olympicstyle weightlifters. J Strength Cond Res, 2003; 17: 746-754

Gabriel YF, Helen KF, Shirley SH, Florence F, Kenneth KK, Desmond CK. Effects of functional weightlifting training on lifting strength and isokinetic peak torque oftrunk and limb muscles.J Physiother Theory Practice, 1998; 14(2): 87-92

García-Pallarés J, López-Gullón JM, Muriel X, Díaz A, Izquierdo M. Physical fitness factors to predict male Olympic wrestling performance. Eur J Appl Physiol, 2011; 111(8): 1747-1758

Golas A, Maszczyk A, Zajac A, Mikolajec K, Stastny P. Optimizing post activation potentiation for explosive activities in competitive sports. J Hum Kinet, 2016; 52: 95-106

Grabiner M, Jeziorowski J. Isokinetic trunk extension and flexion strength-endurance relationships. Clin Biomech, 1991; 6(2): 118-22

Guilhem G, Giroux C,Couturier A, Maffiuletti NA. Validity of trunk extensor and flexor torque measurements using isokinetic dynamometry. J Electromy Kinesiol, 2014; 24(6): 986-93

Guzik DC, Keller TS, Szpalski M, Park JH, Spengler DM. A biomechanical model of the lumbar spine during upright isometric flexion, extension, and lateral bending. Spine, 1996; 21(4): 427-433

Hedrick A. Training the trunk for improved athletic performance. Strength Cond J, 2000; 22(3): 50-61

Henchoz Y, So AKL. Exercise and nonspecific low back pain: A literature review. Joint Bone Spine, 2008; 75: $533-539$ 
Hendrick A, Wada H. Weightlifting movements: do the benefits outweigh the risks? Strength Cond J, 2008; 30(2): $26-34$

Hibbs AE, Thompson KG, French D, Wrigley A, Spears I. Optimizing performance by improving core stability and core strength. Sports Med, 2008; 38(12): 995-1008

Hübner-Woźniak E, Kosmol A, Lutoslawska G, Bem EZ. Anaerobic performance of arms and legs in male and female free style wrestlers. J Sci Med Sport, 2004; 7: 473-480

Iwai K, Okada T, Nakazato K, Fujimoto H, Yamamoto Y, Nakajima H. Sport-specific

characteristics of trunk muscles in collegiate wrestlers and judokas. J Strength Cond Res, 2008; 22(2): 350-8

Jones MA, Stratton G, Reilly T, Unnithan VB. Biological risk indicators for recurrent non-specific low back pain in adolescents. Br J Sports Med, 2005; 39: 137-140

Kibler WB, Press J, Sciascia A. The role of core stability in athletic function. Sports Med, 2006; 36: 189-198

Kolber MJ, Beekhuizen K. Lumbar stabilization: An evidence-based approach for the athlete with low back pain. Strength Cond J, 2007; 29: 26-37

Kraemer WJ, Fry AC, Rubin MR, Triplett-McBride T, Gordon SE, Koziris LP, Lynch JM. Physiological and performance responses to tournament wrestling. Med Sci Sports Exerc, 2001; 33: 1367-1378

Kraemer WJ, Vescovi JD, Dixon P. The physiological basis of wrestling: implications for conditioning programs. Strength Cond J, 2014; 26(2): 10-15

Lalongo C. Understanding the effect size and its measures. Biochemia Medica, 2016; 26(2): 150-63

Lee JH, Hoshino Y, Nakamura K, Kariya Y, Saita K, Ito K. Trunk muscle weakness as a risk factor for low back pain. A 5-year prospective study. Spine, 1999; 24(1): 54

Maszczyk A, Golas A, Czuba M, Krol H, Wilk M, Stastny P, Goodwin J, Kostrzewa M, Zajac A. EMG Analysis and Modelling of Flat Bench Press Using Artificial Neural Networks. SAJRPER. 2016; 38(1): 91-103.

Mirzaei B, Curby DG, Rahmani-Nia F, Moghadasi M. Physiological profile of elite Iranian junior freestyle wrestlers. J Strength Cond Res, 2009; 23(8): 2339-2344

Müller J, Müller S, Stoll J, Fröhlich K, Otto C, Mayer F. Back pain prevalence in adolescent athletes. Scand J Med Sci Sports, 2017; 27: 448-454

Mueller S, Mayer P, Baur H, Mayer F. Higher velocities in isokinetic dynamometry: a pilot study of new test mode with active compensation of inertia. Isokinet Exerc Sci, 2011; 19: 63-70

Murlasits Z. Special considerations for designing wrestling-specific resistance training programs. Strength Cond J, 2004; 26(3): 46-50

Orchard JW, Farhart P, Leopold C. Lumbar spine region pathology and hamstring and calf injuries in athletes: is there a connection? Br J Sports Med, 2004; 38(4): 502-4

Recio JC, López-Plaza D, Barbado Murillo D, García-Vaquero MP, Vera-García FJ. Reliability assessment and correlation analysis of 3 protocols to measure trunk muscle strength and endurance. J Sports Sci, 2017; 36(4): 357-364

Renkawitz T, Boluki D, Grifka J. The association of low back pain, neuromuscular imbalance, and trunk extension strength in athletes. Spine J, 2006; 6: 673-683

Renkawitz T, Linhardt O, Grifka J. Electric efficiency of the erector spinae in high performance amateur tennis players. J Sports Med Phys Fitness, 2008; 48: 409-416

Sassmannshausen G, Smith BG. Back pain in the young athlete. Clin Sports Med, 2002; 21: 121-189

Secchi LLB, Muratt MD, Andrade NVS, Greve JMA. Isokinetic trunk dynamometry in diferent swimming strokes. Acta Ortop Bras, 2010; 18(5): 295-7

Skrzeka A, Ignasiaka Z, Kozieł S, Sławinska T, Rozek K. Differences in muscle strength depend on age, gender, and muscle functions. Isokinet Exerc Sci, 2012; 20: 229-235

Soldati A, Cicchella A, Bombardi F, Gradilone A, Lubich T. Trunk muscle strength in athletes test using a specific isokinetic dynamometer. Medi dello Sport, 1992; 45(3): 339-342 
Stastny P, Gołaś A, Blazek D, Maszczyk A, Wilk M, Pietraszewski P, Petr M, Uhlir P, Zając A. A systematic review of surface electromyography analyses of the bench press movement task. Plos One, 2017; 12(2): $\mathrm{e} 0171632$

Steele J, Bruce-Low S, Smith D, Jessop D, Osborne N. Lumbar kinematic variability during gait in chronic low back pain and associations with pain, disability and isolated lumbar extension strength. Clin Biomech, 2014; 29: 1131-1138

Storey A, Smith HK. Unique aspects of competitive weightlifting: performance, training and physiology. Sports Med, 2012; 42(9): 769-90

Van Damme BB, Stevens VK, Van Tiggelen DE. Velocity of isokinetic trunk exercises influences back muscle recruitment patterns in healthy subjects. J Electromy Kinesiol, 2013; 378(86): 1873-5711

Ward SR, Kim CW, Eng CM, Gottschalk LJ 4th, Tomiya A, Garfin SR, Lieber RL. Architectural analysis and intraoperative measurements demonstrate the unique design of the multifidus for lumbar spine stability. J Bone Joint Surg Am, 2009; 91: 176-185

Yahia A, Jribi S, Ghroubi S, Elleuch M, Baklouti S, Habib Elleuch M. Evaluation of the posture and muscular strength of the trunk and inferior members of patients with chronic lumbar pain. Joint Bone Spine, 2011; 78(3): 291-7

Yaprak Y. The effect of back extension training on back muscle strength and spinal range of motion in young females. Biol Sport, 2013; 30(3): 201-206

\section{Corresponding author:}

\section{David G. Behm}

School of Human Kinetics and Recreation, Memorial University of Newfoundland,

St. John's, Newfoundland and Labrador, Canada

Tel: 709-864-3408

Fax: 709-864-3979

E-mail: dbehm@mun.ca 